\title{
EARLY RESULTS FROM VLT SPHERE: LONG-SLIT SPECTROSCOPY OF 2MASS 0122-2439 B, A YOUNG COMPANION NEAR THE DEUTERIUM BURNING LIMIT**
}

\author{
Sasha Hinkley ${ }^{1}$, Brendan P. Bowler ${ }^{2,8}$, Arthur Vigan ${ }^{3,4}$, Kimberly M. Aller $^{5}$, Michael C. Liu ${ }^{5}$, Dimitri Mawet ${ }^{4,6}$, \\ Elisabeth Matthews ${ }^{1}$, Zahed Wahhaj ${ }^{4}$, Stefan Kraus ${ }^{1}$, Isabelle Baraffe ${ }^{1,7}$, and Gilles Chabrier ${ }^{1,7}$ \\ ${ }^{1}$ Astrophysics Group, University of Exeter, Physics Building, Stocker Road, Exeter EX4 4QL, UK \\ ${ }^{2}$ Division of Geological and Planetary Sciences, California Institute of Technology, 1200 E. California Boulevard, Pasadena, CA 91125, USA \\ ${ }^{3}$ Aix Marseille Université, CNRS, LAM (Laboratoire d'Astrophysique de Marseille) UMR 7326, F-13388 Marseille, France \\ ${ }^{4}$ European Southern Observatory, Alonso de Cordova 3107, Vitacura, Santiago, Chile \\ ${ }^{5}$ Institute of Astronomy, University of Hawaii, 2860 Woodlawn Drive, Honolulu, HI 96822, USA \\ ${ }^{6}$ Department of Astronomy, California Institute of Technology, Mail Code 249-17, 1200 E. California Boulevard, Pasadena, CA 91125, USA \\ 7 CRAL, ENS-Lyon (CNRS UMR 5574), Lyon, France \\ Received 2015 March 18; accepted 2015 April 24; published 2015 May 19
}

\begin{abstract}
We present $0.95-1.80 \mu \mathrm{m}$ spectroscopy of the $\sim 12-27 M_{\text {Jup }}$ companion orbiting the faint $(R \sim 13.6)$, young ( 120 Myr) M-dwarf 2MASS J01225093-2439505 (“2M0122-2439 B”) at 1".5 separation (50 AU). Our coronagraphic long-slit spectroscopy was obtained with the new high contrast imaging platform Very Large Telescope Spectro-Polarimetric High-contrast Exoplanet REsearch (SPHERE) during Science Verification. The unique long-slit capability of SPHERE enables spectral resolution an order of magnitude higher than other extreme AO exoplanet imaging instruments. With a low mass, cool temperature, and very red colors, 2M0122-2439 B occupies a particularly important region of the substellar color-magnitude diagram by bridging the warm directly imaged hot planets with late-M/early-L spectral types (e.g., $\beta$ Pic b and ROXs $42 \mathrm{Bb}$ ) and the cooler, dusty objects near the L/T transition (HR 8799bcde and 2MASS 1207b). We fit BT-Settl atmospheric models to our $R \approx 350$ spectrum and find $T_{\text {eff }}=1600 \pm 100 \mathrm{~K}$ and $\log (g)=4.5 \pm 0.5 \mathrm{dex}$. Visual analysis of our 2M0122-2439 B spectrum suggests a spectral type L3-L4, and we resolve shallow $J$-band alkali lines, confirming its low gravity and youth. Specifically, we use the Allers \& Liu spectral indices to quantitatively measure the strength of the FeH, VO, KI, spectral features, as well as the overall $H$-band shape. Using these indices, along with the visual spectral type analysis, we classify 2M0122-2439 B as an intermediate gravity object with spectral type L3.7 \pm 1.0 .
\end{abstract}

Key words: instrumentation: adaptive optics - instrumentation: spectrographs - planets and satellites: detection techniques: high angular resolution

\section{INTRODUCTION}

The study of objects with masses and temperatures near the transition between the $\mathrm{L}$ and $\mathrm{T}$ spectral types, the so-called "L/T transition objects," has emerged as one of the most interesting and perplexing phenomena of substellar physics (Barman et al. 2011; Baraffe 2014). Indeed, ongoing observations of substellar objects continue to confirm early indications that spectral types for L/T transition objects have a very strong dependence on surface gravity (e.g., Metchev \& Hillenbrand 2006; Liu et al. 2013b). Specifically, for a given effective temperature, low-gravity objects tend to be classified with spectral types earlier than their effective temperatures would naively predict based on older, higher-gravity objects as well as a handful of brown dwarf and planetary mass objects, e.g., G196-3 B (Rebolo et al. 1998), HD203030B (Metchev \& Hillenbrand 2006), 2MASS 0141 (Kirkpatrick et al. 2006), PSO 318-22 (Liu et al. 2013a, 2013b), and WISE 0047 (Gizis et al. 2015). The effect of dust in the atmospheres of these young, low-gravity objects is very likely linked to this phenomenon (e.g., Chabrier et al. 2000; Saumon \& Marley 2008; Marley et al. 2012). However, the small number of young ( $\lesssim 100-200 \mathrm{Myr})$ objects near the $\mathrm{L} / \mathrm{T}$ transition is currently preventing a detailed understanding of this process.

\footnotetext{
* Based on observations made with ESO Telescopes at the La Silla Paranal Observatory Under Program ID 060.A-9381.

${ }^{8}$ Caltech Joint Center for Planetary Astronomy Fellow.
}

Furthermore, even for modern atmospheric models, relatively narrow wavelength coverage for the small number of $\mathrm{L} / \mathrm{T}$ transition objects has led to strong ambiguities in interpretation. Only when data covering multiple, simultaneous bandpasses are obtained can strong constraints be placed on the physical processes present (e.g., Skemer et al. 2012; Ingraham et al. 2014). As an added limitation, much of our knowledge of these low-gravity phenomena in young L-dwarfs comes from a handful of objects: e.g., the directly imaged planets HR 8799 bcde, $\beta$ Pic b, and 2 M1207b with very young ages of 10-30 Myr (e.g., Zuckerman et al. 2011; Mamajek \& Bell 2014), as well as a handful of free-floating brown dwarfs and planetary mass objects e.g., G196-3 B (Rebolo et al. 1998), 2MASS 0141 (Kirkpatrick et al. 2006), PSO 318-22 (Liu et al. 2013a, 2013b), and WISE 0047 (Gizis et al. 2015). A larger sample of objects spanning a wider range of ages is needed to fully disentangle the effects of age (and hence surface gravity) on the spectra of these objects.

A mid-L companion has recently been discovered (Bowler et al. 2013) orbiting the young M3.5V star 2MASS J01225093-2439505 (hereafter "2M0122-2439"), with an estimated mass of $12-27 M_{\text {Jup }}$. The young age of this system $(\sim 120 \mathrm{Myr})$, coupled with the intrinsic faintness of the host star means that even the relatively modest contrast observations of this companion $(\Delta H=6.2)$ were sensitive to planetary mass companions $\left(\sim 5-20 M_{\text {Jup }}\right)$. This relatively nearby $(\sim 36 \mathrm{pc})$ host star, first recognized to possess strong X-ray emission by Riaz et al. (2006), was subsequently assigned membership to 


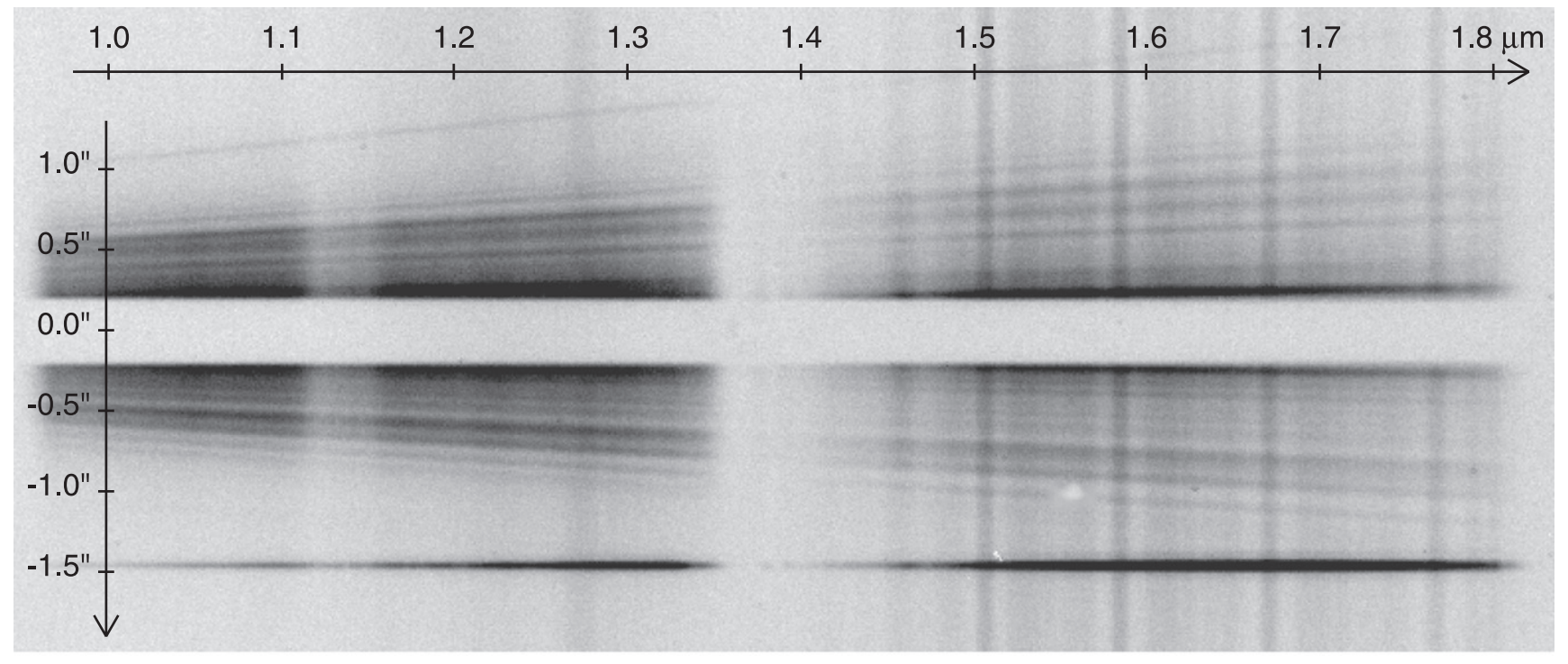

Figure 1. SPHERE-IRDIS medium resolution spectrum of 2M0122-2439 and its companion. The spectrum is dispersed in the horizontal direction, while the vertical direction indicates the spatial direction along the spectrograph slit. The primary is hidden behind the 0."2-radius coronagraphic mask (light horizontal band at the center), so only its halo and speckles are visible in the two-dimensional spectrum. The spectrum of the companion is clearly visible at 1. "' 45 below the primary. Strong $\mathrm{OH}$ sky lines are also visible redward of $1.4 \mu \mathrm{m}$. The top panel shows the 2D spectrum of the primary and companion after combining all the integrations but before any subtraction of the stellar halo and speckles. The lower panel shows the spectrum of the companion after the subtraction detailed in Section 3 .

the 120 Myr AB Dor young moving group (Malo et al. 2013). Since much of our understanding of L-dwarf companions comes from systems with ages $\sim 10-30 \mathrm{Myr}$, the somewhat older age of the 2M0122-2439 system makes it particularly important for mapping out the evolution of giant planets and brown dwarfs. Furthermore, its low temperature coupled with its very red colors make this companion a "bridge" between hot $(\sim 1700-2000 \mathrm{~K})$ directly imaged planets with late-M/early-L spectral type (e.g., ROXs 42Bb and $\beta$ Pic b; Ratzka et al. 2005; Kraus et al. 2014; Currie et al. 2014; Chilcote et al. 2015) and cooler $(\sim 800-1300 \mathrm{~K})$, dusty planetary mass companions (e.g., HR 8799bcde and 2M1207b; Chauvin et al. 2004; Marois et al. 2010).

In this paper, we present near-infrared (NIR) spectroscopy of 2M0122-2439 B using the newly commissioned SpectroPolarimetric High-contrast Exoplanet REsearch (SPHERE) high contrast imaging platform at Very Large Telescope (VLT) obtained during science verification observations. Our observations were obtained using a combination of coronagraphy and long-slit spectroscopy, unique to the SPHERE instrument. In Sections 2 and 3, we describe the observations and analysis. In Section 4, we describe our results of spectral typing and constraints on the surface gravity measurements.

\section{OBSERVATIONS}

Our observations were gathered on UT 2014 December 6 during the Science Verification period of the SPHERE instrument (e.g., Beuzit et al. 2008) at the VLT located at Cerro Paranal in Chile. The data were obtained using the Infrared Dual-band Imager and Spectrograph (IRDIS; Dohlen et al. 2008) instrument configured in the long slit spectroscopy (hereafter "LSS") mode, using the 0"'09 slit providing medium resolution $(R \approx 350)$ across the $Y J H$-bands (Vigan et al. 2008). This observing mode is unique to SPHERE with the coronagraphic mask embedded into the slit to create a long slit coronagraph, enabling a spectral resolution an order of magnitude higher than the integral field spectrographs found in SPHERE and other new high-contrast instruments (e.g., Hinkley et al. 2011; Macintosh et al. 2014).

The observations consisted of 55 coronagraphic observations resulting in a total exposure time of 59 minutes on target. Subsequent to this, a $20 \mathrm{~s}$ observation of HD 10342, an A0V spectroscopic standard placed off the coronagraphic mask, but within the slit, was obtained. No sky backgrounds were acquired. Despite the faintness of the host $\operatorname{star}(R=13.6)$, Strehl ratios of $\sim 70 \%$ were achieved throughout the observations thanks to the SAXO extreme adaptive optics system (Fusco et al. 2013). The DIMM seeing was stable around 1"!0 throughout the first half of the observations, and no seeing data were available for the second half. Wavelength calibration was acquired during the night at the end of the science observations using arc lamps.

\section{DATA REDUCTION AND ANALYSIS}

Standard calibrations were produced using the preliminary release (v0.14.0-2) of the SPHERE Data Reduction and Handling pipeline (Pavlov et al. 2008). The 55 science frames were median combined, producing a two-dimensional spectrum that is shown in the top panel of Figure 1. In addition to the strong signal from the companion, residual, uncorrected quasistatic speckles (e.g., Hinkley et al. 2007; Crepp et al. 2011) can be seen tracing diagonal paths with wavelength.

For the subtraction of the stellar halo and speckles at the position of the companion, we tried two approaches, both 

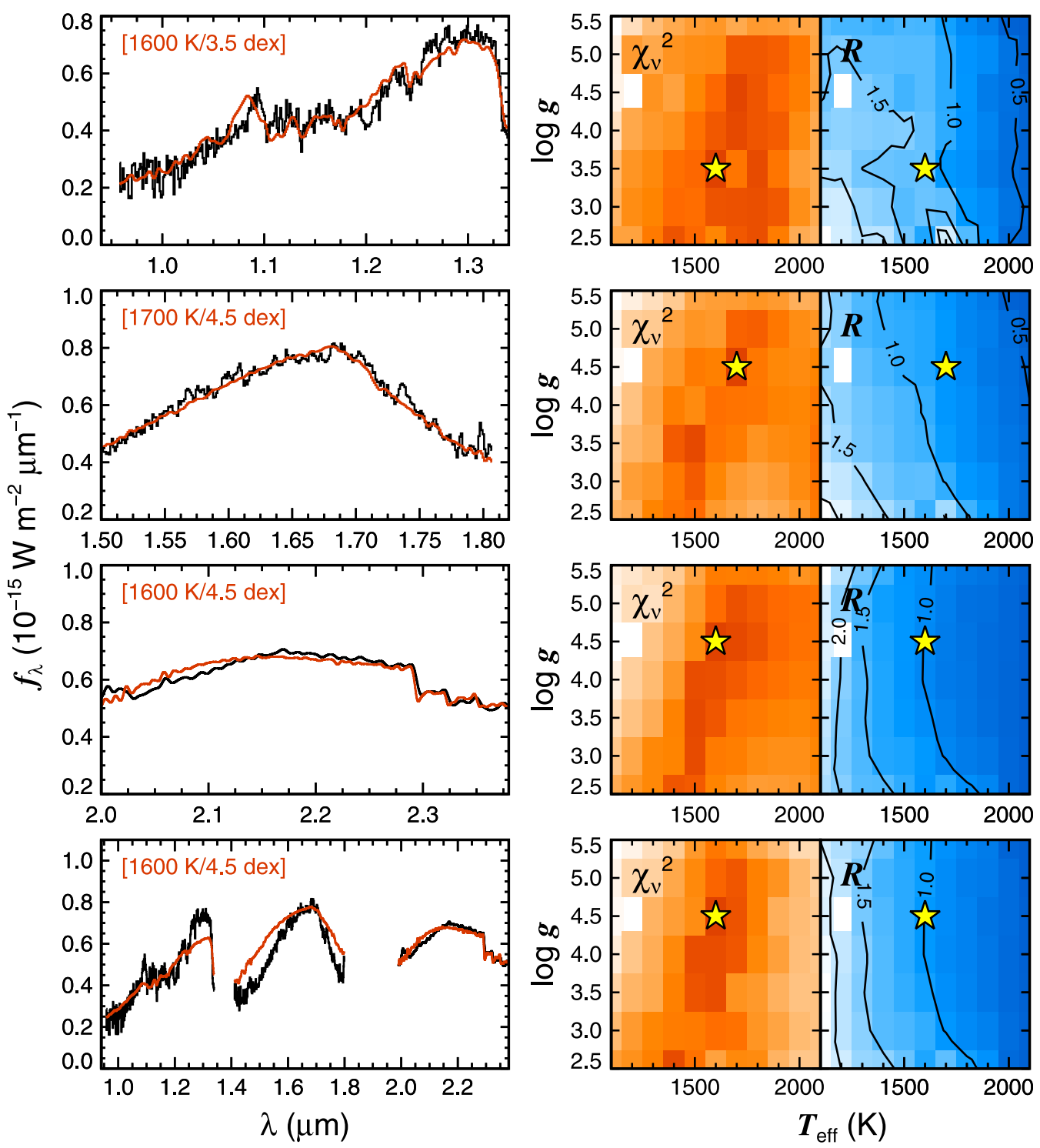

Figure 2. Atmospheric model fits (red) to our new SPHERE 0.9-1.8 $\mu \mathrm{m}$ spectrum of 2M0122-2439 B together with the $K$-band OSIRIS spectrum from Bowler et al. (2013; black). The BT-Settl models from Allard et al. (2011) yield temperatures of 1600-1700 K and a surface gravity of 3.5-4.5 dex among individual band fits and the entire $0.9-2.4 \mu \mathrm{m}$ spectrum. The right panels display reduced $\chi^{2}$ and inferred radius contours (in $R_{\mathrm{Jup}}$ ) assuming a distance of $36 \pm 4 \mathrm{pc}$.

providing similar results. The first approach is similar to spectral differential imaging adapted for LSS data, as presented in Vigan et al. (2008) and demonstrated on-sky in Vigan et al. (2012). The second approach is much simpler as it does not involve any assumption on the spectral dependence of the diffraction features: at the position of the companion, we simply subtracted the diametrically symmetric value of the halo with respect to the star. The validity of this latter approach owes to the faintness of the primary target and companion, which results in the companion being in an area of the spectrum where the data are limited by the photon noise of the halo. Both attempted halo subtraction schemes provide a very good subtraction leaving negligible spectroscopic imprint. The bottom panel of Figure 1 shows the results of this exercise.

The spectrum of the companion and the spectroscopic standard were extracted in the following way: an aperture centered at the object position was created with a width of $\epsilon \lambda / D$ in each spectral channel and the signal was then summed in each channel to obtain a one-dimensional spectrum. The value of $\epsilon$ was varied from 0.5 to 1.5 , but no differences were observed in the output spectrum as a function of the aperture width. The only difference between the extraction of the spectrum of the companion and the spectroscopic standard is the position of the center of the mask in the data. The local noise was estimated by summing the residuals after subtraction on either side of the companion spectrum using the same aperture size as the companion. We verified that the spectrum extracted with the two subtraction schemes and the different aperture sizes lie within these error bars, making us confident that our extraction of the spectrum is correct.

Finally, a telluric correction on the extracted spectrum was performed using the "xtellcor_general" software package (part of the IRTF SpeXtool reduction package; Vacca et al. 2003; Cushing et al. 2004), using the extracted spectrum of the spectroscopic standard star. Despite these wavelength calibration tests, a slight, but uniformly systematic wavelength offset $(0.0025 \mu \mathrm{m})$ persisted in the redward direction. To compensate for this, we manually shifted our spectra by this amount. This 
offset was determined by performing a cross correlation with the 2M0122-2439 B spectra with an L6 brown dwarf obtained from the IRTF library. Such offsets in the final reduced spectra can naturally arise due to non-perfect centering of the faint companion point-spread function in the slit. Comparison of the $H$-band spectrum from Keck/OSIRIS (Bowler et al. 2013) with the $H$-band spectrum from SPHERE shows excellent agreement.

\section{RESULTS}

\subsection{Physical Properties}

We performed model fits to our VLT SPHERE 0.9-1.8 $\mu \mathrm{m}$ spectrum together with the Keck/OSIRIS $K$-band spectrum from Bowler et al. (2013) to infer the physical properties of 2M0122-2439 B. We fit the solar metallicity "CIFIST2011bc" version of the BT-Settl models of Allard et al. (2011) with no $\alpha$-element enhancement to individual $Y+J(0.9-1.35 \mu \mathrm{m}), H$ $(1.5-1.8 \mu \mathrm{m})$, and $K(2.0-2.4 \mu \mathrm{m})$ bands as well as the full $0.9-2.4 \mu \mathrm{m}$ spectrum. The models are first Gaussian smoothed to the same resolution as the SPHERE data $(R \approx 350)$ and interpolated onto the wavelength sampling of our spectrum. Our analysis is carried out in a Monte Carlo fashion incorporating spectral measurement errors and band-to-band flux calibration uncertainties as described in Bowler et al. (2011). Briefly, for each trial we generate a new spectrum by randomly adding Gaussian noise drawn from spectral errors at each pixel. Likewise, a new flux calibration scale factor is drawn from a Gaussian distribution for each trial based on the measured photometry and uncertainties of 2M0122-2439 B. Note that only two flux calibration scale factors are considered here: one for our SPHERE $0.9-1.8 \mu \mathrm{m}$ spectrum and one for the OSIRIS $K$-band spectrum. Following Cushing et al. (2005) we compute a model scale factor, equal to $R^{2} / d^{2}$, and a reduced $\chi^{2}$ value for each BT-Settl model spanning $T_{\text {eff }}=1100-2100 \mathrm{~K}\left(\Delta T_{\text {eff }}\right.$ $=100 \mathrm{~K})$ and $\log (g)=2.5-5.5 \operatorname{dex}(\Delta \log (g)=0.5 \mathrm{dex})$.

Figure 2 shows the best-fit models, the median reduced $\chi^{2}$ contour maps from 1000 Monte Carlo trials, and the median inferred radius contour map based on the model scale factor and distance estimate of $36 \pm 4 \mathrm{pc}$. The $(1600 \mathrm{~K} / 4.5 \mathrm{dex})$ model provides the best-fit to the full $0.9-2.4 \mu \mathrm{m}$ spectral regions and yields a radius of $1.0 R_{\text {Jup }}$. The $H$-band yields a slightly warmer model $(1700 \mathrm{~K} / 4.5 \mathrm{dex})$ and smaller radius $\left(0.8 R_{\mathrm{Jup}}\right)$, while the $Y J$-band fit yields a slightly lower gravity model $(\log (g)=3.5$ dex $)$ with a slightly larger radius $\left(1.2 R_{\mathrm{Jup}}\right)$. The Monte Carlo procedure produces uncertainties in the temperature and gravity of $100 \mathrm{~K}$ and $0.5 \mathrm{dex}$, respectively. These best fit temperature values are somewhat warmer than the effective temperature predicted by evolutionary models, which ranges from 1350 to $1500 \mathrm{~K}$ (Bowler et al. 2013). Possible reasons for this discrepancy could be imperfections in opacity sources, line lists, and treatment of dust. Also, as Bowler et al. (2013) emphasize, this target is unusually red, possibly exacerbating the $0.95-2.35 \mu \mathrm{m}$ fit. It is also worth noting that the covariance in the $\log (g) / T_{\text {eff }}$ planes shown in Figure 2 also have low $\chi^{2}$ values.

\subsection{Spectral Type and Luminosity}

We determined the NIR spectral type of 2M0122-2439 B using both the index-based ("quantitative") and visual methods described in Allers \& Liu (2013). For consistency with the gravity analysis (Section 4.3), we used the spectrum smoothed
Table 1

Spectral Type and Gravity Indices

\begin{tabular}{lc}
\hline \hline & Spectral Type Indices $^{\mathrm{a}}$ \\
\hline Visual SpT $(J$-band $)$ & $\mathrm{L} 4 \pm 1$ \\
Visual SpT $(K$-band $)$ & $\mathrm{L} 3 \pm 1$ \\
$\mathrm{H}_{2} \mathrm{O}$ & $\ldots$ \\
$\mathrm{H}_{2} \mathrm{O}-\mathrm{D}$ & $\ldots$ \\
$\mathrm{H}_{2} \mathrm{O}-1$ & $14.1_{-1.1}^{+1.1}$ \\
$\mathrm{H}_{2} \mathrm{O}-2$ & $\ldots$ \\
Final Index-based SpT & $14.1_{-1.1}^{+1.1}$ \\
Final SpT & $\mathrm{L} 3.7 \pm 1$ \\
\hline
\end{tabular}

Gravity Indices and Classification $^{\mathrm{b}}$

$\mathrm{FeH}_{z}$

$\mathrm{VO}_{z}$

$\mathrm{KI}_{J}$

H-cont

Overall Gravity Scores

Gravity Class

$1.13_{-0.03}^{+0.03}$
$1.057_{-0.011}^{+0.010}$
$1.095_{-0.003}^{+0.003}$
$0.942_{-0.004}^{+0.004}$
$2011(20 ? ?)$
INT-G

a "Final index-based SpT" is the median SpT calculated from the indices of the Monte Carlo realizations of the spectrum. "Final SpT" refers to the weighted mean of the visual SpT and the index-based SpT.

${ }^{b}$ The gravity score in parenthesis refer to the Allers \& Liu (2013) classification scheme. Objects with index values corresponding to INT-G but are within $1 \sigma$ of the FLD-G value are classified with a score of "?."

to the same resolution $(R \approx 150)$ for the index-based spectral typing. The index-based method combines the spectral-type sensitive indices from Allers et al. (2007), Slesnick et al. (2004), and McLean et al. (2003) to calculate the spectral type. In our case, the $\mathrm{H}_{2} \mathrm{O}-\mathrm{D}$ (McLean et al. 2003) index is not applicable because our spectrum does not include the wavelengths used for the index. Also, the $\mathrm{H}_{2} \mathrm{O}$ (Allers et al. 2007) index is not valid because the spectral types calculated for this index ( $>\mathrm{L} 4)$ are always out of the valid range (M5-L4). Although all the other indices are valid for L dwarfs, they are not valid across the entire spectral class. The $\mathrm{H}_{2} \mathrm{O}-1$ and $\mathrm{H}_{2} \mathrm{O}-2$ (Slesnick et al. 2004) indices are valid for spectral types of M4-L5 and M4-L2, respectively. As such, only the $\mathrm{H}_{2} \mathrm{O}-1$ index is applicable for this object, leading to an index-based SpT of 14.1 \pm 1.1 (See Table 1).

Second, we also visually compared $2 \mathrm{M} 0122-2439 \mathrm{~B}$ to L dwarf very low-gravity (VL-G) and intermediate gravity (INT-G) spectroscopic standards defined in Allers \& Liu (2013). We used standard spectra taken from the IRTF Spectral Library (Cushing et al. 2005) and the SpeX Prism Library (http://pono. ucsd.edu/ adam/browndwarfs/spexprism). Following the visual classification methods for young and intermediate age objects of Allers \& Liu (2013), we normalize both our candidates and the standard template in each NIR band separately (see Figure 3 for an example). We also smoothed our spectrum to $R \approx 130$ in order to compare with the publicly available $\mathrm{L}$ dwarf spectroscopic standards, which were taken at a similar resolution. Since the sequence presented in Figure 3 is a mixture of VL-G and INT-G gravity classes, it should not be treated as a proper Spectral Type sequence, but is still useful for comparison. We assumed an uncertainty of \pm 1 subtype for our visual classification in both the $J$ and $K$-band (consistent with Allers \& Liu 2013). Using this visual comparison method, we classify $2 \mathrm{M} 0122-2439 \mathrm{~B}$ as L4.0 \pm 1.0 in the $J$-band and 

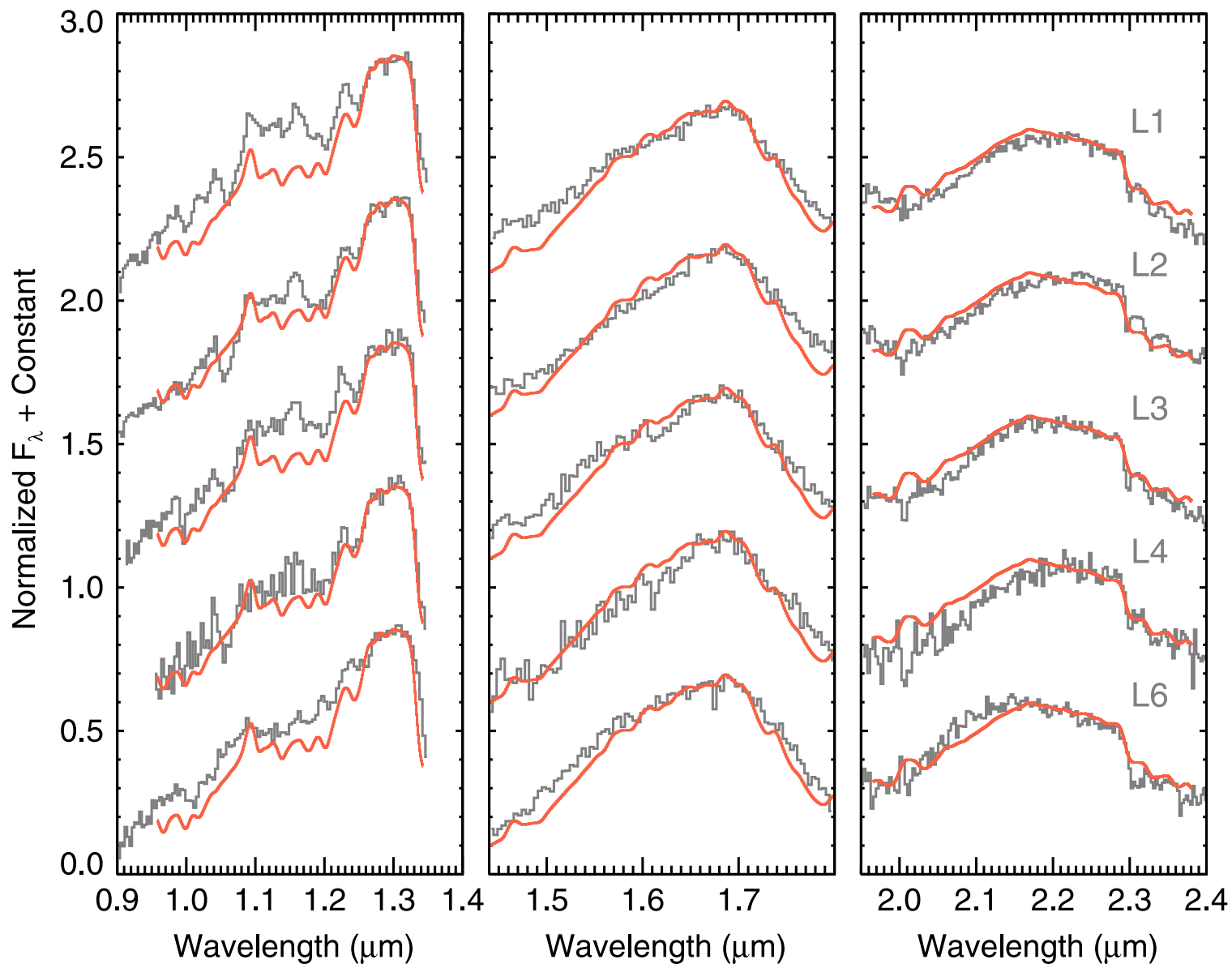

Figure 3. Visual spectral type classification of $2 \mathrm{M} 0122-2439 \mathrm{~B}$ (dark orange) smoothed to $R \approx 130$ (to match the resolution of our comparison spectra) compared with a collection of L dwarf VL-G and INT-G objects taken from Allers \& Liu (2013). The standards are, from top to bottom: 2M0117-34 (L1), 2M0536-19 (L2), $2 \mathrm{M} 1726+25$ (L3), 2M1551+09 (L4), and 2M0103+19 (L6). We classify 2M0122-2439 B as an L4 \pm 1 in the $J$-band and L3 \pm 1 in the $K$-band using this visual comparison.

$\mathrm{L} 3.0 \pm 1.0$ in the $K$-band. The final spectral type is calculated following Allers \& Liu (2013) and is the weighted mean of each of the visual and index-based spectral types, with an adopted uncertainty of 1 subtype. This gives a final spectral type of $\mathrm{L} 3.7 \pm 1.0$.

A bolometric luminosity of 2M0122-2439 B is calculated by integrating the flux-calibrated model spectrum derived in Section 4.1 between wavelengths of 0.25 and $1000 \mu \mathrm{m}$. This gives a value of $\log \left(L / L_{\odot}\right)=-4.16 \pm 0.10 \mathrm{dex}$ with the uncertainty dominated by uncertainty in distance determination. This is in good agreement with the $\log \left(L / L_{\odot}\right)$ $=-4.19 \pm 0.10$ dex cited in Bowler et al. (2013).

\subsection{Gravity Classification}

Young ultracool dwarfs have lower surface gravity than field objects, resulting in distinctive features in their NIR spectra (e.g., Allers et al. 2007; Allers \& Liu 2013). Low resolution $(R \approx 35)$ spectroscopy (e.g., Hinkley et al. 2008) can place strong constraints on the effective temperature, but constraints on surface gravity are more challenging (e.g., Hinkley et al. 2013), requiring higher resolution. At higher spectral resolution, young objects tend to have weaker $\mathrm{FeH}$ and alkali line absorption and stronger $\mathrm{VO}$ absorption in the $J$-band, in addition to a triangular-shaped $H$-band (see Figure 4, upper panels). We compared these spectral features of
2M0122-2439 B with known old, field gravity (FLD-G) and young, VL-G dwarfs of the same spectral type to visually assess the surface gravity (Figure 4, upper panels). For this visual comparison, we smoothed the spectrum of 2M0122-2439 B to $R \approx 130$ to match the native resolution of our comparison spectra. 2M0122-2439 B has weak $\mathrm{FeH}_{z}$, slightly weak $\mathrm{KI}_{J}$ absorption, and a triangular-shaped $H$-band, all signatures of low gravity. However, the $\mathrm{VO}_{z}$ absorption is weak, which is to be expected for this SpT (Allers \& Liu 2013, specifically their Figure 20). We also examined the alkali lines in the $J$ band, which are resolved at the native spectral resolution of $R \approx 350$ (Figure 4, lower panel). 2M0122-2439 B may have slightly weak KI $[1.253 \mu \mathrm{m}]$ and $\mathrm{FeH}_{J}$ lines similar to the VL-G comparison spectrum. However, the NaI $[1.140 \mu \mathrm{m}]$, KI $[1.169 \mu \mathrm{m}]$ and $\mathrm{KI}[1.177 \mu \mathrm{m}]$ lines have too low signal-tonoise to place meaningful constraints on the surface gravity. Overall, the spectral features of 2M0122-2439 B suggest that it has a low surface gravity.

We then quantitatively assessed the gravity classification of our objects using spectral indices from Allers \& Liu (2013). Under this classification scheme, indices are measured in the $J$ and $H$-bands and are each then assigned a score $(0,1$, or 2$)$ according to the index value and the spectral type of the object, with higher numbers indicating lower gravity. However, index values corresponding to INT-G but within $1 \sigma$ of the FLD-G value 

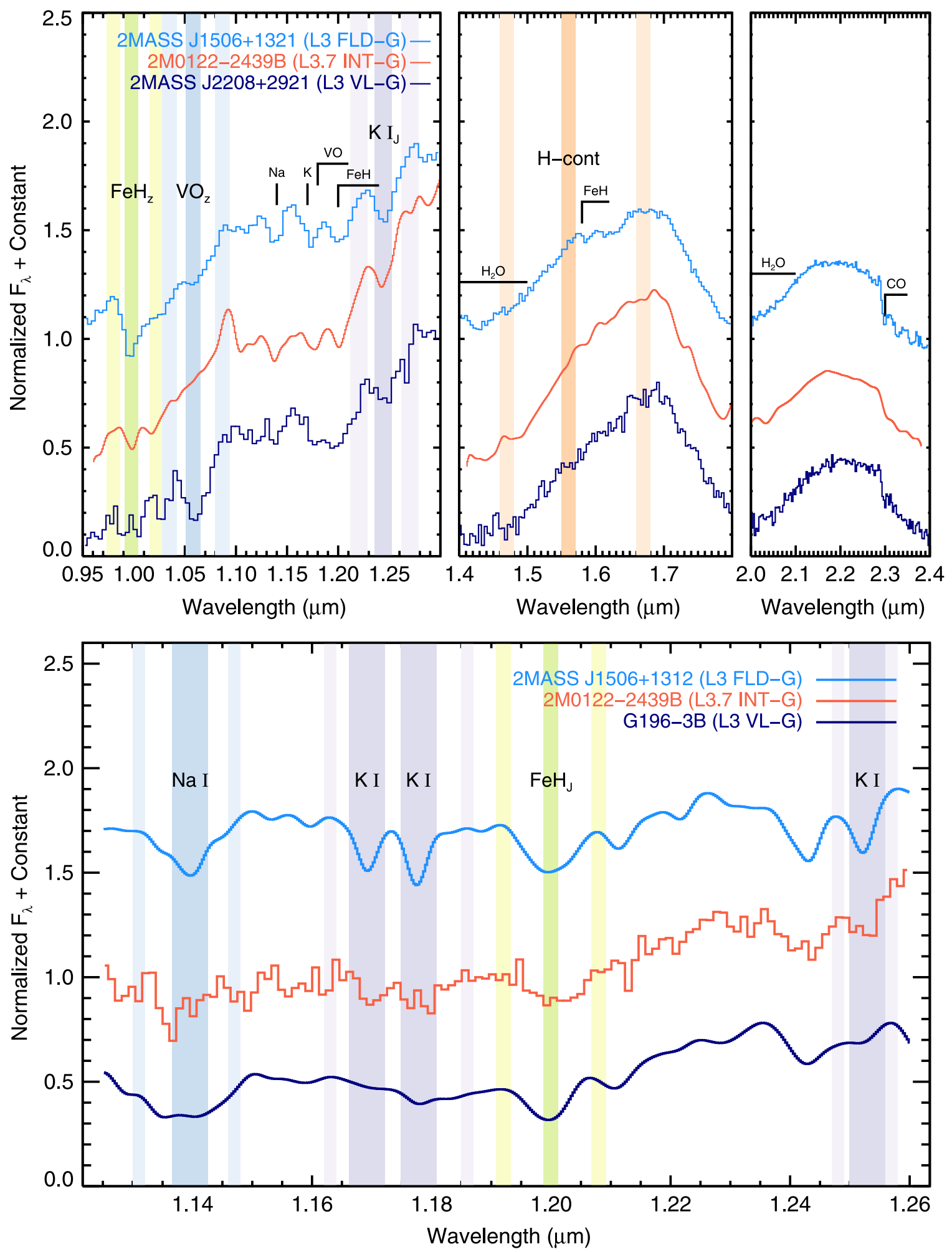

Figure 4. Top panels: NIR spectrum from VLT/SPHERE (YJH-bands) and Keck/OSIRIS ( $K$ band; Bowler et al. 2013) of 2M0122-2439 B (red), compared with a field/FLD-G (light blue) and young/VL-G (dark blue) spectral standard of similar spectral type. For comparison spectra, we used the field standard (FLD-G) and the very low gravity (VL-G) spectral standards from Kirkpatrick et al. (2010) and Allers \& Liu (2013), respectively. In the top panels, we have smoothed our spectrum of $2 \mathrm{M} 0122-2439 \mathrm{~B}$ to $R \approx 130$ to match the resolution of the comparison spectra. Gravity-sensitive features from Allers \& Liu (2013) are labeled and the wavelength ranges used to calculate gravity indices are highlighted for $\mathrm{FeH}_{z}$ (yellow-green), $\mathrm{VO}_{z}$ (blue), $\mathrm{KI}_{J}$ (purple), and $\mathrm{H}$-cont (orange). Lower panel: the $J$-band spectrum in its native resolution $(R \approx 350)$, along with the well-characterized VL-G dwarf G196-3 B (Rebolo et al. 1998) and the same field object as the top panels. The comparison spectra have been smoothed to the resolution of the SPHERE spectrum. Gravity-sensitive features in the $J$-band from Allers \& Liu (2013) are labeled and the wavelength ranges used to calculate gravity indices are highlighted for $\mathrm{NaI}$ (blue), $\mathrm{KI}$ (purple), and $\mathrm{FeH}_{J}$ (yellow-green), though these are for illustration purposes only since our spectrum of $2 \mathrm{M} 0122-2439 \mathrm{~B}$ is too low resolution to use these gravity indices. 
are classified with a score of "?," instead of 1, and ignored. Indices that are not valid because of either the spectral type and/or the resolution of an object are scored as " $n$." These scores are combined into a final four-number gravity score that represents the $\mathrm{FeH}, \mathrm{VO}$, alkali lines, and $\mathrm{H}$-band continuum indices (e.g., 0110, 2110, etc.). Finally, this gravity score is used to determine the overall gravity classification for the object: FLD-G, INT-G, or VL-G.

We also used a modified classification method (Aller et al. 2015), which uses a Monte Carlo simulation to propagate measurement uncertainties into the overall gravity classification uncertainties. However, unlike Allers \& Liu (2013) this method does not give a score of "?" for indices with values corresponding to INT-G but within $1 \sigma$ of the FLD-G values. Instead, this method gives them a score of 1 and thus does not ignore the score. The overall gravity classification value is the median from the values calculated in the Monte Carlo realizations. This allows us to better flag borderline objects between INT-G and FLD-G.

Our spectrum of 2M0122-2439 B is too low resolution $(R \approx 350)$ to use the alkali line indices from Allers \& Liu (2013) (e.g., Figure 4, lower panel) in the $J$ band which require at least $R \approx 750$. Therefore, we assessed the gravity classification of 2M0122-2439 B using the Allers \& Liu (2013) low resolution gravity indices: $\mathrm{FeH}_{z}, \mathrm{VO}_{z}, \mathrm{KI}_{J}$, and $\mathrm{H}-$ cont (Table 1). To calculate these indices, we followed Allers $\&$ Liu (2013) and smoothed the spectrum to $R \approx 150$, because the indices are tailored for that resolution. Our final gravity classification of 2M0122-2439 B is INT-G.

\section{SUMMARY AND CONCLUSIONS}

Using a novel combination of LSS and coronagraphy with the VLT-SPHERE instrument, we have obtained $R \approx 350$ spectra of the young, early/mid L-dwarf 2M0122-2439 B. Atmospheric model fits to our spectra suggest a surface gravity and temperature $T_{\text {eff }}=1600 \pm 100 \mathrm{~K}$ and $\log (g)=4.5 \pm$ 0.5 dex. We also resolve shallow $J$-band alkali lines in 2M0122-2439 B, and use the spectral indices defined in Allers \& Liu (2013) to measure the strength of the FeH, VO, KI, spectral features, as well as the overall $H$-band shape. Using these indices, we confirm the low gravity and youth of 2M0122-2439 B. Visual classification alone suggests a spectral type L3-L4, and the index-based classification scheme outlined in Allers \& Liu (2013), suggests a L4.1 \pm 1.1 spectral type. Combining both of these methods yields a final spectral type of $\mathrm{L} 3.7 \pm 1$.

We thank the anonymous referee for several useful comments. The research of K.M.A. was supported by the National Science Foundation Graduate Research Fellowship under grant No. DGE-1329626. Any opinion, findings, and conclusions or recommendations expressed in this material are those of the authors' and do not necessarily reflect the views of the National Science Foundation. S.K. acknowledges support from an STFC Ernest Rutherford Fellowship ST/J004030/1, Ernest Rutherford grant (ST/K003445/1), and Marie Curie CIG grant (SH-06192). This work is partly supported by Royal
Society award WM090065 and the consolidated STFC grant ST/J001627/1. The research leading to these results has received funding from the European Research Council under the European Community's Seventh Framework Programme (FP7/2007-2013 grant agreement No. 247060).

\section{REFERENCES}

Allard, F., Homeier, D., \& Freytag, B. 2011, in ASP Conf. Ser. 448, XVI Cambridge Workshop on Cool Stars, Stellar Systems, and the Sun, ed. C. Johns-Krull, M. K. Browning, \& A. A. West (San Francisco, CA: ASP), 91

Aller, K. M., Liu, M. C., Magnier, E. A., et al. 2015, ApJ, submitted

Allers, K. N., Jaffe, D. T., Luhman, K. L., et al. 2007, ApJ, 657, 511

Allers, K. N., \& Liu, M. C. 2013, ApJ, 772, 79

Baraffe, I. 2014, in 50 Years of Brown Dwarfs, Astrophysics and Space Science Library, Vol. 401, ed. V. Joergens (Cham, Switzerland: Springer International Publishing), 141

Barman, T. S., Macintosh, B., Konopacky, Q. M., \& Marois, C. 2011, ApJ, 733, 65

Beuzit, J.-L., Feldt, M., Dohlen, K., et al. 2008, Proc. SPIE, 7014, 701418

Bowler, B. P., Liu, M. C., Kraus, A. L., Mann, A. W., \& Ireland, M. J. 2011, ApJ, 743, 148

Bowler, B. P., Liu, M. C., Shkolnik, E. L., \& Dupuy, T. J. 2013, ApJ, 774, 55

Chabrier, G., Baraffe, I., Allard, F., \& Hauschildt, P. 2000, ApJ, 542, 464

Chauvin, G., Lagrange, A., Dumas, C., et al. 2004, A\&A, 425, L29

Chilcote, J., Barman, T., Fitzgerald, M. P., et al. 2015, ApJL, 798, L3

Crepp, J. R., Pueyo, L., Brenner, D., et al. 2011, ApJ, 729, 132

Currie, T., Daemgen, S., Debes, J., et al. 2014, ApJL, 780, L30

Cushing, M. C., Rayner, J. T., \& Vacca, W. D. 2005, ApJ, 623, 1115

Cushing, M. C., Vacca, W. D., \& Rayner, J. T. 2004, PASP, 116, 362

Dohlen, K., Langlois, M., Saisse, M., et al. 2008, Proc. SPIE, 7014, 70143L

Fusco, T., Sauvage, J.-F., \& Petit, C. 2013, in Proc. of the Third AO4ELT Conf., ed. S. Esposito \& L. Fini (Florence, Italy: INAF), 69

Gizis, J. E., Allers, K. N., Liu, M. C., et al. 2015, ApJ, 799, 203

Hinkley, S., Oppenheimer, B. R., Brenner, D., et al. 2008, Proc. SPIE, 7015, 701519

Hinkley, S., Oppenheimer, B. R., Soummer, R., et al. 2007, ApJ, 654, 633

Hinkley, S., Oppenheimer, B. R., Zimmerman, N., et al. 2011, PASP, 123, 74

Hinkley, S., Pueyo, L., Faherty, J. K., et al. 2013, ApJ, 779, 153

Ingraham, P., Marley, M. S., Saumon, D., et al. 2014, ApJL, 794, L15

Kirkpatrick, J. D., Barman, T. S., Burgasser, A. J., et al. 2006, ApJ, 639, 1120

Kirkpatrick, J. D., Looper, D. L., Burgasser, A. J., et al. 2010, ApJS, 190, 100

Kraus, A. L., Ireland, M. J., Cieza, L. A., et al. 2014, ApJ, 781, 20

Liu, M. C., Dupuy, T. J., \& Allers, K. N. 2013a, AN, 334, 85

Liu, M. C., Magnier, E. A., Deacon, N. R., et al. 2013b, ApJL, 777, L20

Macintosh, B., Graham, J. R., Ingraham, P., et al. 2014, PNAS, 111, 12661

Malo, L., Doyon, R., Lafrenière, D., et al. 2013, ApJ, 762, 88

Mamajek, E. E., \& Bell, C. P. M. 2014, MNRAS, 445, 2169

Marley, M. S., Saumon, D., Cushing, M., et al. 2012, ApJ, 754, 135

Marois, C., Zuckerman, B., Konopacky, Q. M., Macintosh, B., \& Barman, T. 2010, Natur, 468, 1080

McLean, I. S., McGovern, M. R., Burgasser, A. J., et al. 2003, ApJ, 596, 561 Metchev, S. A., \& Hillenbrand, L. A. 2006, ApJ, 651, 1166

Pavlov, A., Möller-Nilsson, O., Feldt, M., et al. 2008, Proc. SPIE, 7019, 701939

Ratzka, T., Köhler, R., Leinert, Ch., et al. 2005, A\&A, 437, 611

Rebolo, R., Zapatero Osorio, M. R., Madruga, S., et al. 1998, Sci, 282, 1309

Riaz, B., Gizis, J. E., \& Harvin, J. 2006, AJ, 132, 866

Saumon, D., \& Marley, M. S. 2008, ApJ, 689, 1327

Skemer, A. J., Hinz, P. M., Esposito, S., et al. 2012, ApJ, 753, 14

Slesnick, C. L., Hillenbrand, L. A., \& Carpenter, J. M. 2004, ApJ, 610, 1045

Vacca, W. D., Cushing, M. C., \& Rayner, J. T. 2003, PASP, 115, 389

Vigan, A., Bonnefoy, M., Chauvin, G., Moutou, C., \& Montagnier, G. 2012, A\&A, 540, A131

Vigan, A., Langlois, M., Moutou, C., \& Dohlen, K. 2008, A\&A, 489, 1345

Zuckerman, B., Rhee, J. H., Song, I., \& Bessell, M. S. 2011, ApJ, 732, 61 